Revue d'histoire de l'Amérique française

\title{
L'affaire Daigle contre Tremblay : le temps comme moteur du débat social au Québec à la fin des années 1980
}

\section{Marilyne Caouette}

Volume 72, numéro 3, hiver 2019

URI : https://id.erudit.org/iderudit/1059981ar

DOI : https://doi.org/10.7202/1059981ar

Aller au sommaire du numéro

Éditeur(s)

Institut d'histoire de l’Amérique française

ISSN

0035-2357 (imprimé)

1492-1383 (numérique)

Découvrir la revue

Citer cet article

Caouette, M. (2019). L'affaire Daigle contre Tremblay : le temps comme moteur du débat social au Québec à la fin des années 1980. Revue d'histoire de l’Amérique française, 72(3), 73-95. https://doi.org/10.7202/1059981ar
Résumé de l'article

Décriminalisé depuis 1988, l'avortement demeure pourtant une pratique controversée. À l'été 1989, l'affaire Daigle contre Tremblay déclenche un débat public qui soulève dans l'espace médiatique un vaste ensemble d'arguments et de considérations rattachés à l'avortement. Entre le 17 juillet et le 8 août, Chantal Daigle et Jean-Guy Tremblay s'affrontent en Cour afin de déterminer si la jeune femme peut recourir à un avortement après que Jean-Guy Tremblay, père du futur enfant, eut requis une injonction pour l'en empêcher. L'affaire ayant été amplement médiatisée, les journaux québécois sont principalement mis à profit pour dresser une étude de cas et rendre compte des discours d'une grande variété d'intervenants, à commencer par les groupes pro-vie et pro-choix. Cet article montre que la dynamique des multiples intervenants provoque un sentiment d'urgence qui teinte l'ensemble du débat et le transforme en affaire de société. À une époque où les relations entre les hommes et les femmes sont en pleine transformation, l'affaire permet d'assister au déploiement d'une pensée conservatrice au Québec, mais aussi d'une forte riposte contre ce backlash par plusieurs groupes.
Tous droits réservés @ Institut d’histoire de l’Amérique française, 2019
Ce document est protégé par la loi sur le droit d'auteur. L'utilisation des services d'Érudit (y compris la reproduction) est assujettie à sa politique d'utilisation que vous pouvez consulter en ligne.

https://apropos.erudit.org/fr/usagers/politique-dutilisation/ 


\title{
le temps comme moteur du débat social au Québec à la fin des années $1980^{2}$
}

\author{
Marilyne CaOuette \\ Université du Québec à Trois-Rivières
}

RÉSUMÉ • Décriminalisé depuis 1988, l'avortement demeure pourtant une pratique controversée. À l'été 1989, l'affaire Daigle contre Tremblay déclenche un débat public qui soulève dans l'espace médiatique un vaste ensemble d'arguments et de considérations rattachés à l'avortement. Entre le 17 juillet et le 8 août, Chantal Daigle et Jean-Guy Tremblay s'affrontent en Cour afin de déterminer si la jeune femme peut recourir à un avortement après que Jean-Guy Tremblay, père du futur enfant, eut requis une injonction pour l'en empêcher. L'affaire ayant été amplement médiatisée, les journaux québécois sont principalement mis à profit pour dresser une étude de cas et rendre compte des discours d'une grande variété d'intervenants, à commencer par les groupes pro-vie et pro-choix. Cet article montre que la dynamique des multiples intervenants provoque un sentiment d'urgence qui teinte l'ensemble du débat et le transforme en affaire de société. À une époque où les relations entre les hommes et les femmes sont en pleine transformation, l'affaire permet d'assister au déploiement d'une pensée conservatrice au Québec, mais aussi d'une forte riposte contre ce backlash par plusieurs groupes.

1. Lorsque Jean-Guy Tremblay obtient une injonction à la Cour, l'affaire se nomme Tremblay contre Daigle. Toutefois, Chantal Daigle choisit de poursuivre les procédures judiciaires et selon la façon dont nous abordons cette affaire, c'est elle qui continue cette bataille juridique afin de faire renverser le jugement de la Cour supérieure sur l'injonction. Nous avons donc choisi d'utiliser Daigle contre Tremblay pour désigner l'affaire.

2. J'aimerais remercier tout spécialement Marise Bachand pour son soutien et son aide. Merci également aux évaluateurs de mon mémoire de maitrise, Peter Gossage et Thierry Nootens, et aux évaluateurs anonymes de la Revue, pour leurs commentaires et leurs réflexions qui ont su enrichir grandement cet article. Leur contribution a été essentielle pour se rendre à la publication. 
ABSTRACT - Although decriminalized since 1988, abortion remains a controversial practice. In the summer of 1989, the Daigle vs Tremblay case triggered a public debate that raised a wide set of arguments and considerations about abortion in the media. Between July 17 and August 8, Chantal Daigle and Jean-Guy Tremblay fought in court to determine whether the young woman could seek an abortion after an injunction was issued by Jean-Guy Tremblay, the father of the future child. Since the events were widely scrutinized by the media, Quebec papers were used to study the discourses of the various parties involved in the debate, especially the pro-life and pro-choice groups. This article shows that the dynamic between the people involved created a sense of urgency that permeated the debate, transforming it into a societal affair. During a time of profound changes in gender relations, this case allowed the emergence of a conservative discourse in Quebec as well as a strong response against such backlash by several groups.

«

Chantal devra garder son bébé. » Ainsi titre en une le Journal de Montréal le 18 juillet 1989. La veille, le juge Jacques Viens de la Cour supérieure de Val-d'Or avait interdit à Chantal Daigle d'avoir recours à un avortement en acceptant la demande d'injonction interlocutoire de Jean-Guy Tremblay, son ancien conjoint. Cette décision représente le point de départ d'une saga judiciaire qui tiendra le Québec en haleine pendant plusieurs semaines. Alors que d'ordinaire, les procédures judiciaires peuvent prendre plusieurs mois, les jugements dans cette affaire seront rendus très rapidement ${ }^{3}$. Le 26 juillet, l'avocat Daniel Bédard plaide la cause de la jeune femme de Chibougamau devant la Cour d'appel, qui confirme la décision de la Cour supérieure à trois juges contre deux. La Cour suprême, le 8 août 1989, annulera finalement l'injonction, après avoir appris que Chantal Daigle s'était fait avorter aux États-Unis. Une dispute privée est ainsi à l'origine d'un grand débat de société sur l'avortement qui trouvera écho jusqu'en Grande-Bretagne ${ }^{4}$. Tout au long de l'affaire, le temps devient moteur du débat social et contribue à créer un sentiment d'urgence.

L'affaire Daigle contre Tremblay survient à une époque où de plus en plus de Québécois se reconnaissent dans le slogan féministe: «Nous aurons

3. Le jugement de la Cour supérieure est rendu le 17 juillet 1989 (Jean-Guy Tremblay $c$. Chantal Daigle, [1989], C.S., no. 170-05-000012-898), celui de la Cour d'appel le 26 juillet (Chantal Daigle $c$. Jean-Guy Tremblay, [1989], C.A., no. 200-09-000393-899) et celui de la Cour suprême le 8 août 1989 (Tremblay c. Daigle, [1989], C.S.C, no. 21553).

4. Chantal Daigle accorde une entrevue exclusive au journal britannique Mail on Sunday. Presse canadienne, "Chantal au Mail on Sunday: "Je ne pouvais pas rester avec un jaloux" ", Le Journal de Montréal, 13 août 1989 , p. 5. 
les enfants que nous voulons 5 .» À la fin des années 1980, les méthodes contraceptives sont courantes et socialement acceptées. Les Québécois sont nombreux à recourir à la planification familiale depuis les années 1960, malgré les réserves de l'Église catholique quant à sa moralité6. Les couples contrôlent mieux leur fécondité qu'auparavant ${ }^{7}$. Ils disposent de ressources comme le Centre de planification familiale du Québec, mis sur pied par le docteur Serge Mongeau en 1967, précédé de l'Association pour la planification familiale de Montréal (APFM) en 1964 et du Service de régulation des naissances Séréna en $1955^{8}$. Les cliniques Lazure, des cliniques de planification familiale où il est possible d'obtenir des avortements thérapeutiques, ont été mises en place dès 1977, mais le service d'avortement y est inégal à travers le Québec ${ }^{9}$ La révolution féministe transforme aussi la manière dont les femmes vivent la maternité en remettant en question plusieurs idées et pratiques sociales traditionnelles, ce qui leur procure davantage de liberté dans le rapport qu'elles entretiennent avec leur propre corps. Les expériences paternelles, quant à elles, sont de plus en plus diversifiées ${ }^{10}$. Dès le début de la décennie 1980, par exemple, la plupart des pères sont présents lors de l'accouchement ${ }^{11}$. Il revient dorénavant à chaque père de définir le type d'expérience qu'il souhaite avoir avec son enfant ${ }^{12}$.

Dans ce contexte de libéralisation de la reproduction, l'avortement demeure cependant controversé. Depuis 1969, la pratique est légalisée grâce à la loi omnibus, adoptée par le parlement canadien ${ }^{13}$. Une femme dont la santé est menacée peut obtenir un avortement avec l'approbation d'un

5. Louise Desmarais, Mémoires d'une bataille inachevée, la lutte pour l'avortement au Québec (1970-1992) (Montréal, Éditions Trait d'union, 1999), p. 146. Le slogan est également le titre de la première pièce (1974) du Théâtre des cuisines, une troupe inspirée du mouvement féministe de la décennie 1970.

6. En 1968, la contraception est officiellement rejetée par Paul VI, qui la qualifie d'immorale. Diane Gervais, Peter Gossage et Danielle Gauvreau, La fécondité des Québécoises, 1870-1970: d'une exception à l'autre (Montréal, Boréal, 2007), p. 250-253.

7. D. Gervais, P. Gossage et D. Gauvreau, La fécondité des Québécoises..., p. 239.

8. D. Gervais, P. Gossage et D. Gauvreau, La fécondité des Québécoises..., p. 261-266.

9. Monica Dunn et al., Focus on Abortion Services in Quebec (Canadian for Choice et la Fédération du Québec pour le planning des naissances, 2010), p. 18.

10. Germain Dulac, La paternité: les transformations sociales récentes (Montréal, Conseil de la famille, 1993), p. 8.

11. Andrée Rivard, De la naissance et des pères (Montréal, Les éditions du remue-ménage, 2016), p. 142.

12. C'est d'ailleurs ce que remarque la sociologue Anne Quiénart dans une recherche qu'elle mène entre 1994 et 1996. Elle conclut que ses résultats "font donc ressortir non pas un modèle de paternité mais bien de multiples façons d'être père... ». Anne Quiénart, «Les formes contemporaines de la paternité au Québec ", dans Nathalie Dyke et Jean-François Saucier, dir., La Paternité aujourd'hui. Bilan et nouvelles recherches, Actes du $66^{\mathrm{e}}$ congrès de l'ACFAS (Québec, Montréal, Université Laval, Centre de recherche et de formation (CRF) du CLSC Côte-des-Neiges, 1998), p. 26.

13. Loi de 1968-1969 modifiant le droit pénal (S.C. 1968-69, c. 38). Cette loi décriminalise également la contraception et l'homosexualité. 
comité thérapeutique. L'accès demeure pourtant difficile, car seule une infime fraction des médecins accepte de pratiquer des avortements ${ }^{14}$. Les médecins peuvent refuser de pratiquer un avortement tout comme les hôpitaux peuvent refuser de mettre en place les comités, ce qui rend l'accès à ce service variable d'une région à l'autre du pays ${ }^{15}$. Ce problème d'accès pousse certains CLSC (Centres locaux de services communautaires), dont le nombre a augmenté au fil des années depuis leur création dans les années 1970, à offrir le service sans mettre en place les comités demandés par la loi ${ }^{16}$. Les Centres de santé des femmes offriront, de leur côté, plus d’accessibilité à ces services aux femmes, dont le premier Centre de santé des femmes de Montréal, créé en $1975^{17}$.

Dans les mois qui précèdent l'affaire Daigle contre Tremblay, les procès du $\mathrm{D}^{\mathrm{r}}$ Henry Morgentaler et de Joseph Borowski sont fortement médiatisés. Alors que le premier est poursuivi pour les avortements illégaux qu'il pratique dans ses cliniques privées, le second choisit de se présenter devant les tribunaux afin que des droits soient attribués au fotus ${ }^{18}$. Pendant plusieurs années, le docteur Morgentaler sera poursuivi devant la justice. En 1976, après l'arrivée au pouvoir du Parti québécois, le ministre de la Justice met fin à toutes les poursuites contre Morgentaler. Cette décision rendra l'avortement réellement léga ${ }^{19}$. D’autres procès seront intentés contre le médecin pendant la décennie 1980, entre autres lorsqu'il tente d'ouvrir une clinique à Toronto en 1983. Au terme de ce procès, en janvier 1988, la Cour suprême décriminalise l'avortement en affirmant l'inconstitutionnalité de l'article 251 du Code criminel. La Cour soutient qu'il va à l'encontre de l'article 7 de la Charte canadienne des droits et libertés qui assure la sécurité de la personne ${ }^{20}$. Toutefois, la décision ne s'applique qu'à la loi en vigueur et laisse ainsi aux gouvernements le droit

14. En 1991, seulement 75 médecins sur 13500 acceptaient de réaliser des avortements. Béatrice Godard, L'avortement, entre la loi et la médecine (Montréal, Liber, 1992), p. 47.

15. B. Godard, L'avortement ..., p. 15.

16. M. Dunn et al., Focus on Abortion..., p. 19-23.

17. M. Dunn et al., Focus on Abortion..., p. 18.

18. Joseph Borowski est un militant pro-vie et ancien ministre manitobain du Nouveau Parti démocratique. Il se présente devant la Cour en 1989 afin de faire reconnaître la personnalité juridique du fotus. La plus haute instance judiciaire du pays choisit de mettre fin au débat en concluant que l'affaire est dorénavant trop hypothétique depuis que la Cour suprême a invalidé l'article 251 du Code criminel dans l'affaire Morgentaler. La Cour statue que le Parlement doit légiférer sur les droits des fotus. Louise Desmarais, La bataille de l'avortement, chronique québécoise (Montréal, Les éditions du remue-ménage, 2016), p. 267.

19. Rachael Elizabeth Grace Johnstone, "The Politics of Abortion in Canada After Morgentaler: Women's Rights as Citizenship Rights ", thèse de doctorat (sciences politiques), Queen’s University, 2012, p. 54.

20. B. Godard, L'avortement, entre la loi et la médecine..., p. 31. 
de recriminaliser l'avortement ${ }^{21}$. Le projet de loi C-45, qui sera proposé par le Parti conservateur mais rejeté par le Sénat en novembre 1989, témoigne de cette possibilité.

Au début de juillet 1989, une Torontoise, Barbara Dodd, se voit interdire par les tribunaux le recours à un avortement, à la demande de son partenaire. Dans ce cas, la Cour d'appel annule le premier jugement. De plus, tout de suite après l'avortement, Dodd déclarera aux journaux que les groupes pro-choix l'ont poussée à commettre un geste qu'elle regrette désormais. La jeune femme soutiendra que ces groupes se sont servis d'elle et qu'ils ne la supportent désormais plus ${ }^{22}$. Les quotidiens montréalais traitent aussi de litiges semblables se déroulant aux États-Unis. Mary Sue Davis et Junior Lewis Davis du Tennessee se retrouvent en procès après leur séparation. La jeune femme souhaite pouvoir décongeler des embryons, alors que son ancien conjoint ne désire plus avoir d'enfant avec elle $^{23}$. Soulevant des enjeux analogues à l'affaire Daigle contre Tremblay, aucun de ces cas ne prend cependant la même ampleur.

L'histoire de Chantal Daigle et de Jean-Guy Tremblay devient l'affaire de tout le Québec: hommes, femmes, médecins, infirmières, politiciens, juristes, journalistes, groupes de pression et d'intérêt. Les groupes de pression pro-vie et pro-choix prennent l'avant-scène d'un débat dont le point central reste l'avortement. Dans cette affaire, les groupes de pression présentent différentes versions de la réalité et tentent de la «déconstruire $»^{24}$. Pendant toute la durée des événements, les groupes de pression se démarquent en tentant non seulement d'influencer les instances du pouvoir, mais également en essayant d'imposer le rythme. Alors que les groupes pro-vie s'efforcent de ralentir les prises de décisions, à l'instar des politiciens au pouvoir, les groupes pro-choix essaient de les accélérer. Comme l'explique Martin Pâquet, certaines parties de la population choisissent la temporalité qui correspond à leurs besoins, plutôt que d'accepter celles des dirigeants lorsqu'elles se rendent compte que cette temporalité contrecarre les objectifs des pressions qu'elles excercent ${ }^{25}$. Le sentiment

21. Jocelyn Downie et Chris Kaposy, "Judicial Reasoning About Pregnancy and Choice», Health Law Journal (2008), vol. 16, p. 303.

22. Suzanne Colpron, "Je vais me battre" - Chantal Daigle», La Presse, 19 juillet 1989, p. A2.

23. Associated Press, "Au Tennessee. Un couple se bat pour décider sur le sort de sept embryons congelés", Le Devoir, 8 août 1989, p. 3.

24. Luc Boltanski et Élisabeth Claverie, "Du monde social en tant que scène d'un procès », dans Luc Boltanski et al., Affaires, scandales et grandes causes (Paris, Stock, 2007), p. 438-439.

25. Martin Pâquet, "Du temps des groupes de pression», dans Stéphane Savard et Jérôme Boivin, dir., De la représentation à la manifestation: Groupes de pression et enjeux politiques au Québec, $19^{e}$ et $20^{e}$ siècles (Québec, Septentrion, 2014), p. 434-435. 
d'urgence rattaché au développement du foetus accélère d'autant plus la dynamique entre les groupes de pression et les politiciens et rythme l'ensemble du débat sur l'avortement à l'été 1989.

Les historiennes et autres spécialistes en études féministes qui se sont intéressées à l'affaire Daigle contre Tremblay l'ont interprétée comme un net recul pour les droits des femmes ${ }^{26}$. Insistant sur la fragilité des acquis féministes, ces travaux se concentrent principalement sur les conséquences de l'affaire, sans s'intéresser de près à son déploiement. Cet article approche l'affaire d'un nouvel angle en examinant sa temporalité. Il montre qu'au fur et à mesure que les divers jugements sont rendus, le rythme s'accélère, le nombre d'intervenants se multiplie, provoquant un débat de société profond qui dépasse la seule question des droits des femmes.

Nos sources principales sont tirées des articles du Devoir, de La Presse et du Journal de Montréal. Ces journaux ont été dépouillés systématiquement du 7 juillet au 17 août 1989 afin d'obtenir une vision globale de l'été 1989, ce qui inclut tous les articles qui se rapportent de manière générale à l'avortement et à la maternité. Plus de 540 articles de journaux ont été retenus, dont 52 premières pages. Près d'une centaine d'articles de journaux anglophones, provenant principalement des quotidiens The Gazette et The Globe and Mail, soit la revue de presse préservée par le groupe Campagne Québec-Vie, font aussi partie du corpus. À l'instar des journaux francophones, les journaux anglophones font des jugements rendus des jalons importants de leur couverture de l'affaire. Les articles en anglais, moins utilisés dans ce texte, rapportaient des idées similaires à celles des principaux quotidiens francophones. À cause des contraintes de temps, nous n'avons pu explorer ces journaux de façon aussi complète que les trois quotidiens francophones, laissant ainsi de côté les caractéristiques particulières de leur couverture tout au long de l'affaire. Nous avons également fait un tour d'horizon de quelques journaux régionaux. Certains quotidiens comme L'écho Abitibien et Le Nouvelliste couvrent l'affaire avec assiduité. Le journal de Trois-Rivières (Le Nouvelliste) accorde une grande place à l'affaire Daigle contre Tremblay, y consacrant huit unes. L'ajout de journaux d'origines

\footnotetext{
26. Micheline de Sève, «Tremblay contre Daigle: retour à l'abc du féminisme », Recherches féministes, 3 , 1 (1990), p. 111-118; Marie-Blanche Tahon, "La mère sans ombre», Recherches féministes, 3, 1 (1990), p. 97-109; Monique Poulin, Le droit à l'avortement et l'affaire Daigle (Québec, Université Laval, groupe de recherche multidisciplinaire féministe, 1996); Diane Lamoureux, Avortement, pratiques, enjeux, contrôle social (Montréal, Les éditions du remue-ménage, 1993) et Denyse Baillargeon, Brève histoire des femmes au Québec (Montréal, Boréal, 2012). La littérature sur l'affaire est largement féministe. Pour une perspective pro-vie, voir Robert Nadeau, "The Anatomy of Evasion: A Critique of Daigle», dans Ian Gentles, dir., A Time to Choose Life: Women, Abortion and Human Rights (Toronto, Éditions Stoddart, 1990), p. 186-200.
} 
géographique et linguistique différentes permet de confirmer plus largement les tendances notées dans les trois quotidiens montréalais. Les médias de masse, de façon générale, présentent les arguments et les actions des groupes de pression qui rejoignent le plus la population et le monde politique $^{27}$. Les journaux, en particulier, permettent de voir quelle importance est accordée aux événements, notamment, grâce aux premières pages ou lorsque plusieurs pages sont consacrées au sujet.

Dans les journaux, les jugements sont des étapes marquantes de l'affaire et structurent cette dernière du début à la fin. Dans les jours précédant et suivant chaque décision, les interventions des groupes de pression sont beaucoup plus nombreuses. La trame narrative que les jugements procurent permet d'expliquer la dynamique entre les multiples intervenants et le sentiment d'urgence qu'elle provoque.

\section{«CHANTAL DAIGLE DEVRA ÊTRE MÈRE »: LE JUGEMENT DE LA COUR SUPÉRIEURE}

Le 17 juillet 1989, Chantal Daigle et Jean-Guy Tremblay se retrouvent en Cour supérieure. Près de deux semaines plus tôt, Chantal Daigle avait décidé de quitter le jeune homme natif de Pointe-aux-Trembles. Après s'être réfugiée chez le propriétaire de son appartement afin d'échapper à son conjoint violent, elle réussit à quitter le domicile qu'ils occupent ${ }^{28}$. Alors qu'elle est en route vers le Centre hospitalier universitaire de Sherbrooke, Daigle apprend qu'une injonction l'empêchant de se faire avorter est émise ${ }^{29}$. La jeune femme choisit de respecter l'ordre de la Cour avec l'intention de s'y présenter pour avoir accès légalement à un avortement.

Le juge Jacques Viens n'autorisera pas la procédure. Il maintient plutôt l'injonction imposée à Chantal Daigle, enceinte de 18 semaines. Selon le juge, le fotus est un être humain. Ce dernier possède donc des droits fondamentaux et les inconvénients causés à Chantal Daigle ne sont pas assez grands pour justifier un avortement. Un des points principaux du débat est ainsi introduit: le fotus est-il un être humain? Le jugement de Jacques Viens attribue une personnalité juridique claire au fotus et des droits au père. Ces droits sont suffisamment considérables pour restreindre les droits des femmes, pourtant déjà reconnus par certains jugements, comme l'arrêt Morgentaler, qui décriminalise l'avortement ${ }^{30}$. La

27. David S. Meyer et Suzanne Staggenborg, "Opposing Movement Strategies in U.S. Abortion Politics ", Social Movements, Conflicts and Change, 28 (2008), p. 216.

28. Chantal Daigle, Le seul choix, le mien (Montréal, Magazine Sérénité, 1990), p. 48-49.

29. L. Desmarais, Mémoires d'une bataille inachevée..., p. 332.

30. R. c. Morgentaler, [1988], 1 R.C.S., 30. 
Cour suprême du Canada avait alors affirmé qu'une femme ne pouvait être forcée à poursuivre une grossesse puisque cela allait à l'encontre de ses droits ${ }^{31}$. Plusieurs spécialistes notent d'ailleurs que la décision du juge Viens va à l'encontre de l'arrêt ou qu'elle manque simplement de fondements juridiques.

Les valeurs et idées du juge Viens sont rapidement scrutées par Le Devoir et La Presse, sous la plume de Lysiane Gagnon, qui le surnomme "le conquistador de Val-d'Or ${ }^{32}$ ». Les conclusions de Jacques Viens représentent une prise de position nette dans le débat sur l'avortement, ce qui est rare dans les instances juridiques et qui explique également la vivacité des réactions. Déjà, les journaux s'intéressent à l'urgence de la situation dans laquelle se retrouve Chantal Daigle. Le 19 juillet, Le Devoir prévoit les différents délais auxquels Chantal Daigle devra faire face:

Normalement, les délais pour remettre les mémoires à la Cour d'appel sont de 60 et 75 jours. Mais hier [...] on indiquait que l'appel pourrait être entendu assez rapidement, compte tenu de la situation de Mme Daigle. Cette dernière n'aurait que deux jours, à partir d'aujourd'hui, pour subir un avortement au Québec. En effet, après 20 semaines, aucun hôpital québécois ne pratique d'avortement «social»"

Chaque jour est donc critique pour la jeune femme et le foetus qui se développe.

Les groupes pro-choix se rassemblent promptement après le jugement ${ }^{34}$. Ils soutiennent que le droit des femmes à l'autonomie reproductrice est mis en péril. La Coalition québécoise pour l'avortement libre et gratuit (CQDALG), le groupe pro-choix le plus impliqué dans le débat, est déjà présente lors de la décision du 17 juillet. Le groupe s'inquiète de la facilité qu'auront désormais les hommes d'empêcher une femme canadienne d'avoir accès à un avortement. Pour la Fédération des femmes du Québec, c'est une "atteinte grave aux droits des femmes, à leur intégrité physique et morale de même qu'à leur dignité, en conférant au foetus un statut de personnalité juridique ${ }^{35}$ ». Plusieurs groupes de pression s'étonnent que la violence conjugale dont a été victime Chantal Daigle ne fasse pas partie des

31. À propos de l'influence des médias pendant le procès Morgentaler, voir Frederick Lee Morton, Morgentaler v. Borowski: Abortion, the Charter and the Courts (Toronto, McLelland \& Stewart, 1992).

32. Lysiane Gagnon, «Le juge de Val-d’Or», La Presse, 22 juillet 1989, p. B3.

33. Presse Canadienne, "Ce matin en cour d'appel. Le juge Chouinard a le sort de Chantal Daigle entre ses mains", Le Devoir, 19 juillet 1989, p. 8.

34. L. Desmarais, Mémoires d'une bataille inachevée..., p. 335.

35. Bruno Bisson, «La décision du juge Jacques Viens provoque l'indignation chez les groupes de femmes ", La Presse, 19 juillet 1989, p. A8. 
considérations du juge Viens. Selon la Coalition pour l'avortement libre et gratuit, le jugement permettra que les hommes violents utilisent le chantage sur leur partenaire ${ }^{36}$. C'est pourquoi le groupe enjoint indirectement Chantal Daigle à se faire avorter. Dès le 27 juillet, Andrée Côté, porte-parole de la Coalition, affirme dans les journaux: "Que celles qui ne peuvent mener à terme leur grossesse se fassent avorter sans aucune crainte, si tel est leur désir. Le gouvernement n'ira jamais jusqu'à emprisonner une femme qui défiera le jugement de la Cour d'appel ${ }^{37}$. " Contrairement à la grande majorité des groupes de femmes et de groupes féministes, l'Aféas (Association féminine d'éducation et d'action sociale) croit que l'avortement est légitime seulement si la santé de la mère ou du foetus est en danger. Interrogée dans Le Nouvelliste, Marie-Marthe Normand, présidente régionale de l'Aféas, ne croit pas que Chantal Daigle devrait obtenir le droit à l'avortement. Elle ajoute que la brutalité de Jean-Guy Tremblay n'est pas une raison suffisante pour avoir recours à cette procédure ${ }^{38}$.

Le débat se dirige aussi parallèlement vers les droits du fotus. Les groupes pro-vie, dont la principale revendication reste les droits du fotus, se réjouissent des gains récents. Ils soutiennent que le foetus est une personne qui détient des droits malgré qu'il ne soit pas encore né. C'est dans cette optique que les partisans pro-vie, surtout depuis le début des années 1980, associent l'avortement au meurtre. D'ailleurs, ce terme revient à l'occasion dans les discours de groupes pro-vie qui paraissent dans les journaux (l'Association des juristes catholiques du Québec), de représentants catholiques (le cardinal Paul Grégoire, évêque de Montréal) et de groupes de droite à intérêt plus général (R.E.A.L Women). Les

Figure I

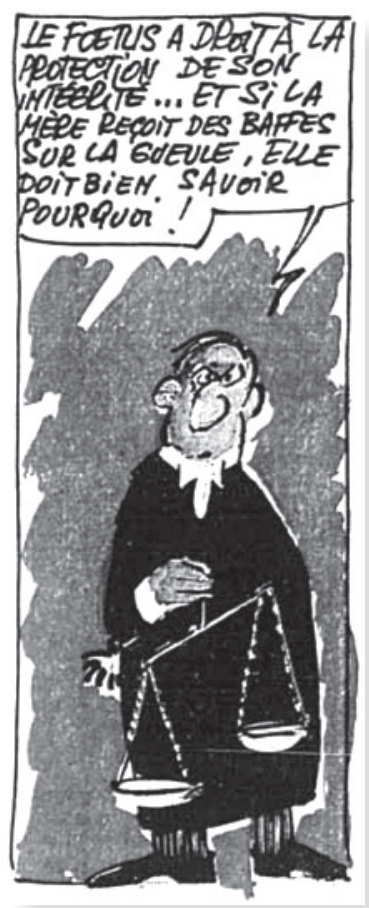

Source: Girerd, La Presse, 23 juillet 1989, p.AI.

36. Bruno Brisson, «Le jugement du juge Viens est fondé sur des articles abolis par la Cour suprême. De l'avis d'un professeur, spécialiste de la question des droits et libertés », La Presse, 18 juillet 1989, p. A2.

37. Martha Gagnon et Josette Brun, «Le jugement de la Cour d’appel sur l'avortement», La Presse, 27 juillet 1989 , p. A5.

38. Doris V. Hamel, «L'Aféas prend position en faveur de l'enfant», Le Nouvelliste, 2 août 1989, p. 6. 
militants pro-vie se réjouissent de chaque jour qui passe sous l'interdiction de l'injonction. Le président de Campagne Québec-Vie, Gilles Grondin, affirme que «[l]e temps travaille pour nous, dans ce cas-là, et il est certain qu'il sera de plus en plus difficile d'éliminer un enfant qui vieillit dans le sein de sa mère ${ }^{39}{ }^{\prime}$. Quelques représentants de l'Église catholique s'expriment sur les droits du foetus, comme le représentant de la Conférence des évêques du Canada et évêque du diocèse de Saint-Jean-Longueuil, Mgr Bernard Hubert, et J. Clément Rainville, prêtre du Grand Séminaire de Montréal, qui exprime l’importance de défendre le fotus. Pour lui, «[1]es droits de l'enfant existent avant l'existence des féministes entêtées ${ }^{40}{ }$. Il présente d'ailleurs une banderole publicitaire qui dit «S.O.S. vive le bébé fœtus» devant le palais de justice. La plupart des intervenants qui se prononcent en faveur des droits du foetus font partie de groupes qui se prononcent uniquement contre l'avortement, alors que ceux privilégiant le droit des femmes incluent aussi des juristes ou des avocats qui interviennent aussi sur d'autres sujets.

Le jugement de la Cour supérieure donne une légitimité à l'action de Jean-Guy Tremblay, mais aussi aux hommes qui ont demandé ce type d'injonction au Canada dans les mois précédents, en affirmant qu'il existe bel et bien des droits du père, soit pendant l'affaire Dodd et dans une cause similaire à Winnipeg. Dans d'autres cas liés à l'avortement, le père est parfois évacué de la question, mais l'injonction demandée par Jean-Guy Tremblay pose la figure du père comme une variable importante de la problématique. Un de ses avocats, Robert Francis, affirme quelques jours après que l'injonction provisoire fut accordée (le 7 juillet), que cette décision enclenche le débat sur les droits du père. En effet, dans sa requête, Jean-Guy Tremblay se base sur ces supposés droits pour justifier son intervention en justice. La place du père est donc mise en valeur au tout début du litige.

On retrouve dans l'importance du rôle du père une manifestation du mouvement masculiniste qui s'installe au Québec à cette époque. Les masculinistes produisent principalement «un discours alarmiste sur la situation des hommes ${ }^{41} »$. Ce mouvement réactionnaire se réapproprie les arguments féministes pour servir la cause des hommes et mettre un frein à l'égalité hommes-femmes. Par exemple, les masculinistes tendent à

39. PR, «La grossesse de Chantal. Une semaine de trop », Le Journal de Montréal, 2 août 1989, p. 5.

40. Presse Canadienne, «Les groupes pro-vie crient victoire», La Presse, 19 juillet 1989, p. A8.

41. Mélissa Blais et Francis Dupuis-Déri, "Qu’est-ce que le masculinisme?", dans Mélissa Blais et Francis Dupuis-Déri, dir., Le mouvement masculiniste au Québec: l'antiféminisme démasqué (Montréal, Les éditions du remue-ménage 2008), p. 13. 
victimiser les hommes en soutenant qu'ils sont désavantagés par rapport aux femmes ${ }^{42}$. Reggie Chartrand, qui prend part au débat entourant Chantal Daigle et Jean-Guy Tremblay en tant que partisan pro-vie, est un parfait exemple des membres de ce mouvement. Dès 1984, il se révolte contre les revendications des femmes pour l'égalité. Selon lui, ces dernières dominent les hommes, qui sont devenus faibles. Indépendantiste convaincu, Chartrand croit que les femmes auraient «castré» les hommes au point où ils seraient dorénavant incapables de réaliser l'indépendance du Québec. Pour Jeffery Vacante, Chartrand "provides one particularly virulent example of this emerging backlash against women ${ }^{43}$ ». Tout comme aux États-Unis, la paternité prend une place importante dans les milieux masculinistes au Québec, ce qui amène la formation de groupes pour les droits des pères.

Lors de l'affaire Daigle contre Tremblay, les groupes pro-vie comme Respect pour la vie et R.E.A.L Women soutiennent Jean-Guy Tremblay et les droits parentaux qu'il invoque. Le président de Campagne Québec-Vie, Gilles Grondin, promet qu'il encouragera d'autres hommes à avoir recours à des injonctions dans les cas où une femme veut avoir un avortement. "Nous allons créer un fouillis juridique», dit-il, en réalisant l'ampleur des événements qui suivent la requête de Tremblay. Quelques jours après le jugement de Jacques Viens, la question des droits du père devient plus superficielle. Il semble y avoir consensus, du moins du point de vue juridique, sur le fait que les droits des pères ne sont pas reconnus par les textes de lois. Alors que pendant plusieurs siècles, le père détenait toute l'autorité dans la famille, des lois ont diminué cette mainmise. En 1970, le Code civil est modifié pour partager l'autorité du père entre les deux parents ${ }^{44}$. La substitution du concept de "puissance paternelle» par celui «d'autorité parentale» participe à l'instauration de plus d'égalité entre les deux parents ${ }^{45}$. Des changements législatifs sont aussi apportés pendant la décennie 1980, par exemple en 1981, lorsque les conjoints deviennent égaux dans le droit de la famille ${ }^{46}$. Toutefois, pour le prêtre du Grand Séminaire de Montréal J. Clément Rainville, «les futurs pères biologiques ont des droits naturels,

42. Martin Dufresne, «Masculinisme et criminalité sexiste», Recherches féministes, 11, 2 (1998), p. 125.

43. Jeffery Vacante, "Liberal Nationalism and the Challenge of Masculinity Studies in Quebec", Left History, 11, 2 (automne 2006), p. 101.

44. Pierre Grelley, "Contrepoints - Le droit des pères", Informations sociales, 2, 176 (2013), p. 23.

45. Peter Gossage, "On Dads and Damages: Looking for the "Priceless Child" and the "Manly Modern” in Quebec's Civil Courts, 1921-1960", Histoire sociale/Social History, 49, 100 (novembre 2016), p. 604.

46. Denyse Baillargeon, Brève histoire des femmes au Québec..., p. 207. 
juridiques et inviolables à faire respecter par tous les citoyens ${ }^{47}$ ». Après le jugement de la Cour d'appel, il est encore moins sûr que juridiquement les pères aient réellement des droits sur le fotus. Le seul juge qui aborde cette question dans sa décision, Yves Bernier, affirme que «c'est sans contredit au père que revient en premier lieu le droit, pour des motifs sérieux et raisonnables, non contraires à l'intérêt de l'enfant à naître, de s'opposer à l'avortement ${ }^{48}$ ".

Quelques jours après que la décision fut rendue, $\mathrm{M}^{\mathrm{e}}$ Daniel Bédard annonce que sa cliente en appellera de la décision. Chantal Daigle souhaite obtenir un nouveau jugement rapidement puisqu'au Québec, la limite pour pratiquer des avortements est fixée à environ vingt semaines. Dans de nombreux articles des trois quotidiens, mais aussi des autres journaux anglophones et régionaux, les auteurs rappellent constamment le nombre de semaines de la grossesse de la jeune femme. Cet accent mis sans relâche sur l'importance de régler la situation rapidement est aussi ressenti par la population. Les questionnements reliés à l'enjeu de l'avortement prennent tout à coup une allure très concrète à travers l'histoire que vivent Chantal Daigle et Jean-Guy Tremblay. Comme le note Donna Greschner, les femmes se sont identifiées à l'histoire de Chantal Daigle: "Women followed every move of Tremblay and the courts, anguished with her, talked amongst ourselves late at night about the pain and horror she must be feeling, concurred with each other that she should ignore the court injunction $^{49}$.» Plusieurs hommes ont aussi eu de la sympathie pour Tremblay, comme en témoignent certains articles de journaux. Le fort sentiment d'urgence transmis par les médias donne une nouvelle perspective à la population qui voit directement l'effet des politiques et des décisions prises par l'État.

\section{«TROIS JUGES ET UN COUFFIN »: LA DÉCISION DE LA COUR D'APPEL}

À 21 semaines de grossesse, Chantal Daigle se voit de nouveau interdire le recours à un avortement. Après seulement cinq jours de délibération, trois juges contre deux confirment le jugement de la Cour supérieure. Ils affirment entre autres que les raisons qui poussent Chantal Daigle à vouloir un avortement ne sont pas suffisantes et qu'un certain droit à la vie

47. YC, "Avortement: l’affaire Daigle entendue dès ce matin », Le Journal de Montréal, 17 juillet 1989, p. 2.

48. Site de la Société québécoise d'information juridique, Jugement de la Cour d'appel, Daigle contre Tremblay, [1989], http://t.soquij.ca/Xd79B, p. 4.

49. Donna Greschner, "Abortion and Democracy for Women: A Critique of Tremblay v. Daigle», McGill Law Journal, 1990, p. 655. 
est reconnu au foetus. Ils considèrent également que l'arrêt Morgentaler de 1988, qui décriminalise l'avortement, n'y donne pas un droit absolu et que leurs conclusions ne vont donc pas à l'encontre de cette décision. Deux juges ont considéré que l'injonction n'était pas supportée par des fondements suffisants ${ }^{50}$.

Dans les jours qui suivent, les journaux couvrent assidument ce jugement dans de nombreux articles recensant les réactions des multiples intervenants. Les textes tiennent aussi fréquemment compte de l'avancement de la grossesse de Chantal Daigle. Le temps presse de plus en plus. L'avocat de Chantal Daigle, Daniel Bédard, insiste sur ce fait et rappelle que cette dernière avait décidé d'avoir recours à un avortement beaucoup plus tôt dans sa grossesse. Il craint que même si la Cour suprême tranche en sa faveur, il soit trop tard. Il affirme qu' "[o]n ne peut dire à Mme Daigle qu'elle a maintenant raison, mais qu'elle ne peut plus se faire avorter parce qu'il est trop $\operatorname{tard}^{51}{ }^{\prime}$. Cette fois, les journalistes ne s'attardent pas sur la personnalité des juges et l'attention se porte davantage vers les conclusions de chacun d'eux.

Les réactions au jugement dépassent le Québec. De groupes comme la Ligue canadienne d'action pour le droit à l'avortement, la Coalition des cliniques d'avortement de l'Ontario et le Comité d'action nationale sur le statut de la femme se prononcent contre le jugement en appel. La porteparole du comité, Judy Rebick, s'indigne que les tribunaux aient le pouvoir de rendre ce genre de décision et dans ce cas particulier, de «traiter les femmes comme des meubles ${ }^{52}$ ». Clayton Ruby, l'avocat de Barbara Dodd, critique lui aussi la décision de la Cour d'appel puisqu'elle relègue les femmes à des citoyennes de seconde zone. Il se moque d'ailleurs du Québec en affirmant: "Quebec is looking more and more like a banana republic ${ }^{53}$.»

Les groupes pro-choix, qui sortent perdants de ce jugement, organisent une manifestation le 27 juillet à Montréal. Les membres de la CQDALG invitent les manifestants et manifestantes à se munir de cintres et d'aiguilles à tricoter afin de rappeler ce que les femmes ont dû subir et les droits obtenus pour lesquels elles ont déjà milité. Plusieurs personnalités

50. Site de la Soquij, Jugement de la Cour d'appel, Daigle contre Tremblay...

51. Jean-Denis Girouard, "(Chantal Daigle: une autre semaine) Justice... », Le Journal de Montréal, 2 août 1989, p. 4.

52. Presse Canadienne, "En 1989, les tribunaux traitent les femmes comme des meubles" - Le comité d'action nationale sur le statut de la femme», La Presse, 3 août 1989, p. A11.

53. Kirk Makin, «Integrity of Judiciary and Rights of Women Dealt Blow, Critics Say », The Globe and Mail, 27 juillet, s.p. 
y prennent part, ainsi que des politiciens. Une nouvelle manifestation le $1^{\mathrm{er}}$ août, organisée, cette fois, par la CQDALG, voit des manifestants provie contre-manifester ${ }^{54}$.

Les groupes de pression pro-vie voient leurs revendications confirmées de nouveau, cette fois par la plus haute cour du Québec. Gilles Grondin, président de Campagne Québec-Vie, affirme être heureux que «[1]es droits du plus faible soient enfin reconnus ${ }^{55}$ ». Bien que les droits du foetus ne soient pas défendus de façon aussi catégorique que dans le jugement de la Cour supérieure, le jugement de la Cour d'appel accorde tout de même au foetus un droit à la vie suffisamment important pour limiter le droit des femmes à l'avortement. En attendant le jugement de la Cour suprême, Henri Kelada, l'avocat de Jean-Guy Tremblay, se réjouit discrètement du temps qui passe. Pour lui, cette attente garde le fotus en vie. «Ils peuvent rendre leur décision en septembre», dit-il avec un sourire ${ }^{56}$. Jean-Guy Tremblay ajoute "[j]e ne l'ai pas réalisé tout de suite, mais je constate que la décision de la Cour d'appel de prendre le temps nécessaire est une victoire pour $\mathrm{moi}^{57}$ ».

Les politiciens, plutôt discrets lors du jugement de la Cour supérieure, prennent d'assaut les journaux au moment où l'affaire prend de l'ampleur. Alors même que la cause est entendue en Cour d'appel, la journaliste Marie Tison, de La Presse, rencontre plusieurs députés conservateurs ${ }^{58}$. Ces derniers affichent une position anti-avortement qui, si elle n'est pas tranchée chez tous les intervenants (principalement les femmes), représente tout de même une tendance pro-vie qui se fera sentir au moment du vote pour une nouvelle loi.

Au fédéral, les députés libéraux et néo-démocrates attribuent directement la responsabilité des événements au Parti conservateur au pouvoir, qui n’a pas su combler le vide juridique ${ }^{59}$. Le Parti conservateur confirme qu'il tentera de proposer une loi sur l'avortement, mais il ne peut assurer ou même prédire si cette loi assouplira l'accès à l'avortement ou l'encadrera davantage. À aucun moment le premier ministre du Canada, Brian

54. Une manifestation est aussi organisée par d'autres groupes pro-choix le 3 août 1989 à Toronto devant la Cour provinciale. Bruno Bisson, « 500 personnes manifestent pour l'avortement», La Presse, 2 août 1989, p. A5.

55. Martha Gagnon, «Les droits du plus faible enfin reconnus», La Presse, 28 juillet 1989, p. A5.

56. André Pépin, «Chantal Daigle attendra la décision de la Cour», 21 juillet 1989, p. A2.

57. A. Pépin, "Chantal Daigle attendra...», p. A2.

58. Marie Tison, «Avortement: les députés du PC veulent une loi rapidement», La Presse, 26 juillet 1989, p. B1.

59. Guy Taillefer, «L'opposition à Ottawa blâme l'incurie du gouvernement Mulroney», La Presse, 27 juillet 1989, p. A4 
Mulroney, ne se risque à commenter l'affaire. Bien que la majorité des questions lui soient adressées, il ne croit pas qu'il devrait commenter une affaire en cours. Il explique son silence par son respect pour le travail des tribunaux. Selon lui, il serait «inapproprié $[\ldots]$ de s'immiscer dans le processus judiciaire ${ }^{60}$ ». L'attentisme de Brian Mulroney ne passe pas inaperçu comme le démontre le titre de cet article de The Gazette: «Mulroney Plays Possum on Abortion ${ }^{61} »$. Le premier ministre tente de temporiser et de faire baisser la pression, ce que lui permettent les délibérations des différentes cours. Malgré cela, les pressions s'accentuent. Au provincial, c'est le chef du Parti québécois, Jacques Parizeau, qui donne du fil à retordre aux politiciens au pouvoir. Dès le 19 juillet, soit deux jours après le jugement de la Cour supérieure, Parizeau déclare qu'il est urgent que le gouvernement fédéral légifère sur l'avortement. Il note entre autres, à l’instar de Judy Rebick, que le vide juridique donne un trop grand pouvoir aux juges ${ }^{62}$.

Depuis le jugement de la Cour suprême de 1988 dans l'affaire Morgentaler, la loi sur les comités thérapeutiques a été invalidée et l'avortement décriminalisé. Le gouvernement conservateur avait tenu au printemps 1988 un vote libre qui n'a pas permis d'en arriver à un consensus. Le projet de loi a ensuite été abandonné ${ }^{63}$. Le jugement de 1988 est donc la dernière décision sur l'avortement. Pour tous les partis, le vide juridique semble poser problème. Les politiciens paraissent convaincus de l'importance d'une nouvelle loi, mais très incertains de son contenu ${ }^{64}$. Ils débattent également afin de déterminer à qui revient la responsabilité de combler ce vide. D’autres intervenants se prononcent sur le besoin d'une législation. J. Clément Rainville, par exemple, croit que le vide juridique facilite trop les avortements ${ }^{65}$.

Après le jugement de la Cour d'appel, Parizeau dénonce cette nouvelle décision majoritaire des juges. Cette fois, il exige que le gouvernement provincial légifère. Il soutient que le plus récent jugement interpelle mainp. 6.

60. Presse Canadienne, "Nous allons agir” - Brian Mulroney », Le Journal de Montréal, 27 juillet 1989,

61. William F. Gold, «Mulroney Plays Possum on Abortion», The Gazette, s.d, s.p.

62. Presse Canadienne (Ottawa), "À la suite du jugement Viens, "Ottawa doit se brancher" », La Presse, 19 juillet 1989, p. B1.

63. Marie Tison, «Le gouvernement fédéral reste indécis», La Presse, 19 juillet 1989, p. B1.

64. Alors que cette idée fait consensus à l'époque, Louise Langevin soutient qu'il est faux de dire que l'absence de loi sur l'avortement crée un vide juridique. En effet, elle affirme plutôt que l'absence d'une loi ne pose pas de réel problème puisque dans notre système démocratique, il n’est pas nécessaire qu'une loi soit édictée pour permettre une action quelconque. Louise Langevin, «Entre la non-reconnaissance et la protection: la situation juridique de l'embryon et du foetus au Canada et au Québec», Revue internationale de droit comparé, 56, 1 (2004), p. 48.

65. Claire Harting, " "Le retour à la dignité" - Émile Colas », Le Journal de Montréal, 19 juillet 1989, p. 11. 
tenant le gouvernement provincial puisqu'il traite de la Charte québécoise des droits et libertés et du Code civil. Le gouvernement québécois a donc lui aussi une responsabilité dans l'encadrement de la pratique; ce qu'il peut faire en précisant la définition d'une personne humaine dans la Charte québécoise des droits et libertés ${ }^{66}$. Il ajoute plus tard que cette tâche reviendra au provincial puisque le fédéral ne semble pas vouloir agir ${ }^{67}$. Le gouvernement libéral au Québec est très peu présent dans les journaux et manque à l'appel lorsqu'il est question de prendre position sur l'un ou l'autre des sujets. Une fois l'affaire conclue, la journaliste Francine Pelletier critiquera d'ailleurs le gouvernement québécois pour avoir consacré son énergie à discuter du partage des compétences plutôt que de tenter de réparer les torts faits aux femmes. Elle écrira: «[p]lutôt que de rectifier l'entorse faite au système judiciaire et aux libertés civiles, plutôt que de réparer l'outrage fait aux femmes, il se lance, fort de son nouveau nationalisme tout de fleur-de-lys vêtu, dans une bataille "de compétences" vis-à-vis [sic] Ottawa ${ }^{68}$."

En effet, à travers le débat sur la législation se développe une autre discussion concernant les responsabilités de chaque gouvernement. Les intervenants attribuent la responsabilité au gouvernement provincial ou fédéral de légiférer le plus tôt possible sur l'avortement. La plupart des politiciens estiment qu'il est urgent de présenter une loi et, après le jugement de la Cour d'appel, les libéraux et les néo-démocrates joignent leurs voix pour presser le gouvernement de Brian Mulroney de le faire.

Pendant les années 1980, le Canada tente de définir le fédéralisme canadien plus clairement, entre autres avec la nouvelle constitution de 1982. En revanche, des embûches se présentent dans ses relations avec le Québec, comme en témoignent le référendum de 1980 ou l'échec de l'Accord du lac Meech. Le Canada travaille donc à se définir davantage et l'affaire Daigle contre Tremblay permet aux politiciens de promouvoir leur vision du partage de certaines compétences. Après le jugement de la Cour d'appel, le procureur général du Canada, Doug Lewis, demande à la Cour suprême d'être entendu et de pouvoir déposer un mémoire lors de l'audience. Lewis y soutient que la Cour d'appel a fait erreur. Les juges ne pouvaient pas restreindre l'accès à l'avortement de Chantal Daigle puisque ce droit relève

66. Paule des Rivières, «Parizeau somme Québec de légiférer», Le Devoir, 28 juillet, p. 14.

67. Presse Canadienne, «Jacques Parizeau croit que c'est à Québec de combler le vide juridique», $L a$ Presse, 29 juillet 1989, p. B3.

68. Francine Pelletier, «En se rendant justice, Chantal Daigle a rendu justice à toutes les femmes», La Presse, 12 août 1989, p. B2. 
du gouvernement fédéral ${ }^{69}$. Plus loin, il précise qu'aucune interprétation des lois ne devrait mener à l'interdiction de l'avortement ${ }^{70}$. Gil Rémillard, alors procureur général du Québec, dépose aussi un mémoire à la Cour suprême en réponse à celui du procureur général du Canada. Il plaide que la Cour d'appel n'a pas empiété sur la compétence législative fédérale lors de son jugement du 26 juillet $^{71}$. Selon ce qu'il affirme dans La Presse, le jugement de la Cour d’appel inclut dorénavant le gouvernement québécois dans le débat sur l'avortement en raison des nombreuses références au Code civil $^{72}$. Gil Rémillard somme d'ailleurs le gouvernement fédéral de prendre ses responsabilités et de combler le vide juridique, ce que lui seul peut réellement faire ${ }^{73}$. La discussion est donc élargie par les politiciens qui désirent utiliser l'affaire Daigle contre Tremblay pour faire avancer leurs propres programmes.

\section{«COUP DE THÉÂTRE À LA COUR... »: LA COUR SUPRÊME DONNE RAISON À CHANTAL DAIGLE}

Le jugement de la Cour suprême du Canada est rendu dans un climat agité. Alors que les juges, qui étaient quelques jours plus tôt en vacances, sont en train de délibérer, on leur apprend que Chantal Daigle a eu recours à un avortement aux États-Unis. Les trois journaux accordent la une à la nouvelle. Gilles Paquin de La Presse parle de l'avortement de Chantal Daigle comme d'une "catastrophe» et d'un "coup de théâtre »" . Le Devoir, sous la plume de Jean- $V$. Dufresne, note une "consternation générale » à la Cour après que l'annonce de l'avortement de Chantal Daigle eut été faite aux juges "médusés »" aussi dans un article du Journal de Montréal ${ }^{76}$. L'avortement de Chantal Daigle éclipse presque la décision des juges, qui n’ont pas encore publié leurs motifs. On retrouve un traitement identique dans Le Nouvelliste.

69. Bbliothèque et Archives nationales du Québec (BANQ), V-M, P644, 1989, mémoire du procureur général du Québec, p. 2.

70. Marie Tison, «L'affaire Chantal Daigle. Québec dépose son mémoire à la Cour», Le Journal de Montréal, 4 août 1989, p. 68.

71. BANQ, V-M, P644, 1989, mémoire du procureur général du Québec, p. 2.

72. Denis Lessard, "Une décision "prudente et réaliste”, selon le gouvernement du Québec. "Stupéfiant", commente Jacques Parizeau ", La Presse, 27 juillet 1989, p. A4.

73. Normand Girard, "Avortement: un mémoire de Québec à la Cour suprême», Le Journal de Montréal, 3 août 1989, p. 11.

74. Gilles Paquin, «La Cour suprême casse l’injonction même si Chantal Daigle s'est déjà fait avorter ", La Presse, 9 août 1989, p. A1.

75. Jean-V. Dufresne, «Les juges donnent raison à Chantal Daigle. Coup de théâtre à la Cour: l'avortement a déjà eu lieu ", Le Devoir, 9 août 1989, p. 1.

76. Presse Canadienne, "Chantal n'a pas attendu la cour. L'injonction a été cassée par le plus haut tribunal », Le Journal de Montréal, 9 août 1989, p. 3. 
Figure 2

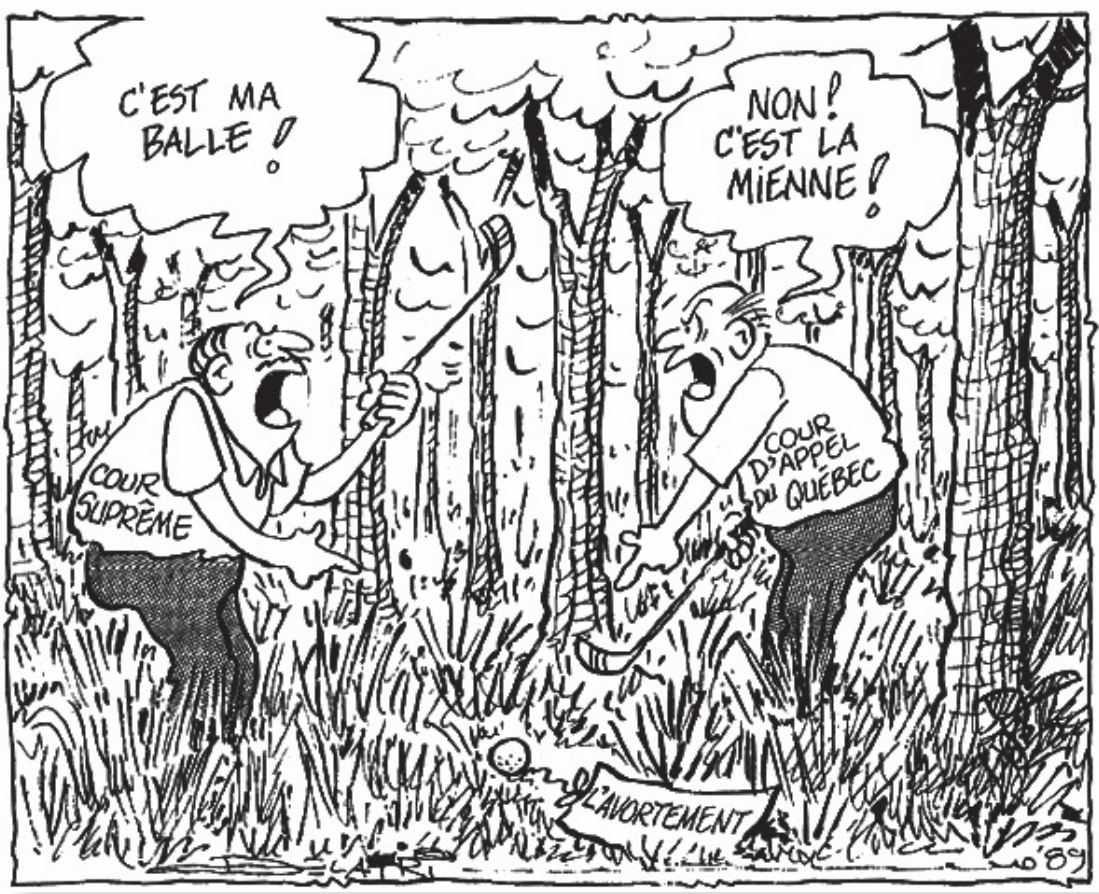

Source; Belatri, Le Nouvelliste, 7 août 1989, p. 6.

Alors que de nombreux groupes pro-choix (incluant des groupes féministes, des spécialistes en droit et autres groupes qui se rallient à la cause de Chantal Daigle) font des droits des femmes l'enjeu principal du débat, ce jugement relègue ces droits au second rang. Comme le soulignent les juges, le but est plutôt de savoir si les droits invoqués par l'intimé - soit les droits du foetus et du père - soutiennent réellement l'injonction émise ${ }^{77}$. Les droits du père sont rapidement discrédités et selon les juges, ni le Code civil ni la Charte canadienne des droits et libertés ne confèrent une personnalité juridique au foetus. Malgré le manque d'affirmation de droit pour les femmes, le jugement représente un précédent judiciaire favorable au droit à l'avortement. La décision de la Cour ignore cependant l'argument de Chantal Daigle selon lequel elle a été victime de violence conjugale. Cela constitue un autre type de précédent, qui prévient les femmes que la

77. Jugement de la Cour suprême, Tremblay contre Daigle [1989], site de l'Institut canadien d'information juridique, https:/ / www.canlii.org/fr/ca/csc/doc/1989/1989canlii33/1989canlii33.html, page consultée le 22 octobre 2014, p. 18. 
violence conjugale ne constitue par un argument juridique solide $\mathrm{e}^{78}$. De plus, le jugement ne comble pas le vide juridique que plusieurs dénoncent. La Cour renvoie plutôt la responsabilité de légiférer sur les droits du foetus au parlement.

Après le jugement de la Cour suprême, le débat se transforme en conversation, en réflexion. La grossesse de Chantal Daigle, à la base du sentiment d'urgence qui a enflammé le Québec, est maintenant terminée. Le Devoir s'attarde le moins à l'affaire après sa conclusion. Le Nouvelliste, un quotidien régional qui a offert une couverture complète de l'affaire, passe encore plus brusquement à autre chose. La Presse et Le Journal de Montréal s'attardent davantage à discuter des différentes conclusions à tirer de cette histoire. La Presse publie des articles qui se concentrent sur les deux anciens conjoints, puis des articles récapitulatifs qui proposent une réflexion sur l'affaire. De façon similaire, le Journal de Montréal se concentre sur les réactions des groupes de pression, de la famille de Chantal Daigle, des politiciens et de la population. Les émotions de Chantal Daigle expliquent pour certains cette décision de recourir clandestinement à un avortement. Selon le Dr Paul Auclair, porte-parole du groupe Médecins pour le respect pour la vie, Chantal Daigle a «certainement un peu paniqué, perdu la tête ${ }^{79}$ ». Jacques Parizeau en fait la même interprétation lorsqu'il soutient que la constante attention médiatique a sans doute joué dans la réaction de la jeune femme, qui l'aurait difficilement supportée $e^{80}$. Les groupes pro-vie essuient un revers important, alors que la plus haute Cour du pays renie les gains obtenus grâce aux deux jugements des cours du Québec. Les instances judiciaires ne sont dorénavant plus réceptives à leurs revendications.

Dans les jours suivants, plusieurs articles du Journal de Montréal traitent de la façon dont Jean-Guy Tremblay a vécu cet épisode. Ces articles au ton empathique à l'égard de Tremblay tiennent compte de son point de vue. L'article d'André Ménard du 12 août raconte le congédiement de Jean-Guy Tremblay, alors qu'un autre article relate les difficultés qu'a rencontrées le jeune homme, qui a eu «toutes les misères du monde » à faire émettre l'injonction ${ }^{81}$. Le 10 août, le Journal de Montréal publie un

78. R. E. G. Johnstone, «The Politics of Abortion in Canada After Morgentaler...», p. 127.

79. J. Mouchon, La politique sous l'influence..., p. 43-45.

80. JGM, "Il faut que les politiciens prennent leur responsabilités" - Jacques Parizeau», Le Journal de Montréal, 9 août 1989, p. 2.

81. André Ménard, «...et son employeur qui le congédie. Jean-Guy Tremblay abreuvé d’insultes pendant qu'il discute avec Michel Girouard», Le Journal de Montréal, 12 août 1989, p. 3; FD, «Des problèmes pour signifier l'injonction ", Le Journal de Montréal, 10 août 1989, p. 3. 
long article sur les réactions de Jean-Guy Tremblay après le jugement. François Dowd, qui écrira aussi à propos de Tremblay plus tard en août, décrit le jeune homme comme étant "aigri et bouleversé ${ }^{82}$ ». Alors que Jean-Guy Tremblay raconte son histoire, l'auteur ajoute : «Aussi bien vous le dire tout de suite, c'est une banale histoire de jalousie qui est à l'origine de ce qui allait devenir une première dans les annales judiciaires du Canada $^{83}$.» À l'instar de plusieurs groupes de pression pro-vie, du juge Viens et de trois juges de la Cour d’appel, le journaliste évacue et discrédite la question de la violence conjugale.

\section{CONCLUSION}

Dans une entrevue accordée à Radio-Canada, et diffusée le 13 août 1989, Chantal Daigle exprime son souhait de retrouver sa vie privée: «Les gens vont finir par oublier. Dans combien de temps, je ne le sais pas. J'ai hâte de pouvoir marcher libre dans la rue ${ }^{84}$.» Bien que l'affaire soit terminée et que le sentiment d'urgence se soit estompé, les journaux ont continué de relater l'histoire de Chantal Daigle et de Jean-Guy Tremblay. La vie de ce dernier alimente les quotidiens jusqu'aux années 2000 en raison de nombreuses condamnations pour violence conjugale. Des articles récapitulatifs paraissent aussi, par exemple, lorsque le cas atteint son dixième anniversaire. On $\mathrm{y}$ apprend, par le père de Chantal Daigle, que cette dernière est heureuse et a refait sa vie ${ }^{85}$. Ce même article traite également de l'intérêt du réalisateur Claude Fournier pour l'affaire. Il affirme qu'il tient le scénario d'un film qu'il prépare depuis plusieurs années ${ }^{86}$. Chantal Daigle et Jean-Guy Tremblay n'accordent toutefois jamais d'entrevues. En 2014, l'émission Tout le monde en parlait consacre une demi-heure à l'affaire sur les ondes de la télé de Radio-Canada ${ }^{87}$. Plus récemment, l'émission de radio de la Chaîne IciPremière, Aujourd'hui l'histoire, a diffusé le 26 janvier 2017 un épisode sur l'affaire, commentée par Francine Pelletier.

Les événements de l'été 1989 sont encore examinés aujourd'hui parce qu'ils racontent une histoire dont les thèmes sont toujours débattus. En avril 2013, la clinique d'avortement Morgentaler de Fredericton au Nouveau-

82. François Dowd, “J'étais jaloux. Je n’ai jamais battu Chantal: je l'ai juste brassée” - Jean-Guy Tremblay", Le Journal de Montréal, 10 août 1989, p. 3

83. F. Dowd, “"J’étais jaloux...”", p. 3.

84. Yves Boisvert, "Je suis libre maintenant", Chantal Daigle affirme que le plus dur n’a pas été l'avortement mais de se faire fermer la porte par les juges", La Presse, 14 août 1989, p. A3.

85. Neko Likongo, «Dix ans après », La Presse, 2 août 1999, p. A1.

86. N. Likongo, «Dix ans...», p. A2.

87. Solveig Miller, "Chantale contre Jean-Guy», Tout le monde en parlait, 21m 33, coul. (29 juillet 2014). 
Brunswick, la seule du genre dans la province, ferme ses portes. Les NéoBrunswickoises doivent alors faire une demande pour un avortement dans un hôpital et obtenir l'approbation de deux médecins ou se rendre dans une autre province ${ }^{88}$. En novembre 2014, après plusieurs manifestations relativement à la fermeture de la clinique, le premier ministre acadien Brian Gallant abolit cette obligation du consentement de deux médecins ${ }^{89}$. L'accès à l'avortement est alors facilité. Au mois de novembre 2016, le ministre de la Santé du Québec, Gaétan Barrette, a ajouté au projet de loi 92 une clause interdisant aux manifestants pro-vie de s'approcher à plus de 50 mètres d'une clinique d'avortement $t^{90}$. Trente ans après l'affaire, l'accès des femmes à l'avortement reste fragile.

La couverture que consacre chaque journal à l'affaire Daigle contre Tremblay se révèle particulièrement marquée par les trois jugements qui alimentent les préoccupations formulées dans les quotidiens. La dynamique qui se développe entre les intervenants, surtout au moment des jugements, encadre le débat. Lorsque deux mouvements s'opposent, les «key outcomes» - c'est-à-dire les résultats qui proviennent des pressions - provoquent un changement dans le paysage politique. Cela force les groupes, surtout ceux qui sont désavantagés par la décision, à adapter leurs stratégies $^{11}$. Le jugement Viens fait intensément réagir par ses prises de position fermes sur des sujets controversés. Le jugement de la Cour d'appel renouvelle et amplifie l'agitation chez les groupes de pression, les experts et les politiciens. À chaque jugement, la grossesse de Chantal Daigle se poursuit, et cette situation entretient le sentiment d'urgence. La dynamique entre les intervenants, soit l'insistance de certains politiciens et des groupes pro-choix et la résistance des politiciens au pouvoir et des groupes pro-vie, augmente davantage le rythme effréné de l'affaire et met ainsi au premier plan différents enjeux tels que les droits des femmes, des foetus et des pères, mais également le rôle de l'État et des tribunaux dans l'accès des femmes à l'avortement. Les thèmes ne sont pas nouveaux, mais il devient tout à coup crucial d'en débattre et de leur trouver une solution. Alors que le temps laisse son

88. S.A., Site de Radio-Canada [En ligne], «Fermeture de la clinique d'avortement Morgentaler à Fredericton: une situation déplorée par de nombreux organismes au pays", http:/ /ici.radio-canada.ca/ regions/atlantique/2014/04/11/010-fermeture-clinique-avortement-morgentaler-nb.shtml.

89. S.A., Site de Radio-Canada [En ligne], «Le Nouveau-Brunswick facilite l'accès à l'avortement», http://ici.radio-canada.ca/regions/atlantique/2014/11/26/005-politique-avortement-nouveau-brunswick.shtml.

90. Marco Bélair-Cirino, Site du journal Le Devoir [En ligne], «Barrette repousse les manifestants pro-vie à 50 mètres des cliniques", http://www.ledevoir.com/politique/quebec/485939/ barrette-repousse-les-manifestants-pro-vie-a-50-metres-des-cliniques.

91. D. S. Meyer et S. Staggenborg, "Opposing Movement Strategies...», p. 211-212. 
empreinte sur l'affaire et la transforme en controverse publique, les intervenants favorisent une discussion portant sur une multiplicité de thèmes qui leur tiennent à cœur, ce qui permet d'approfondir le cas. Les politiciens élargissent la discussion au-delà de l'avortement en débattant du partage des compétences entre les niveaux fédéral et provincial. Des institutions plus conservatrices, comme l'Église catholique ou les groupes pro-vie, profitent de l'affaire pour faire valoir leurs positions face aux changements des dernières décennies. Dans une certaine mesure, leurs demandes trouvent un écho favorable auprès des instances politiques et juridiques québécoises. Le mouvement masculiniste tire aussi avantage de la situation pour faire valoir les droits des pères. Ces parties incarnent chacune un aspect du backlash qui prévaut partout en Amérique du Nord et qui rejette les transformations liées à la révolution féministe ${ }^{92}$. Malgré tout, pendant l'affaire Daigle contre Tremblay, ce mouvement de revanche rencontre une contrepartie de taille dans le mouvement féministe québécois, qui met l'accent sur le droit à l'autonomie des femmes sur leur propre corps.

Le 6 juillet 1989, à Winnipeg, le juge Aubrey Hirschfield de la Cour du Banc de la Reine ne donne pas suite à la demande d'injonction de Steven Diamond. Ce dernier souhaitait empêcher une femme d'avorter du foetus dont il était le père présumé ${ }^{93}$. Pour expliquer sa décision, le juge affirmera plus tard que les femmes ont un droit absolu sur leur propre corps ${ }^{94}$. L'injonction imposée à Barbara Dodd par la Cour supérieure de l'Ontario est également rejetée en appel. Dans le cas québécois, la Cour supérieure et la Cour d'appel interdisent à Chantal Daigle d'obtenir un avortement. Ces deux jugements remettent donc en question le droit à l'avortement qui, après sa légalisation et sa décriminalisation, semblait être acquis. C'est la Cour suprême du Canada qui annule finalement l'injonction. Du même coup, elle invalide plusieurs positions prises par les juges québécois sur les droits des fotus, des pères et des femmes. Alors qu'ailleurs au Canada, les causes se sont réglées plutôt rapidement, sans grand tumulte, le cas Daigle contre Tremblay déclenche une série d'interrogations.

Comme l'expliquent les historiens Nicolas Offenstadt et Stéphane Van Damme, une affaire qui prend de l'importance et de l'intensité remet souvent en question des valeurs, des croyances et des "catégories»,

92. Susan Faludi, Backlash: la guerre froide contre les femmes (Paris, Éditions des femmes, 1993).

93. L. Desmarais, Mémoires d'une bataille inachevée..., p. 332.

94. Presse Canadienne, «Barbara Dodd interjette appel», La Presse, 8 juillet 1989, p. F16. 
lorsqu'elle se développe dans l'espace public ${ }^{95}$. Dans ce cas, la moralité de l'avortement, les conditions dans lesquelles il devrait ou ne devrait pas être pratiqué, les droits de chacun lorsqu'il en est question: tout semble être reconsidéré. Au passage, d'autres questions primordiales doivent être posées. Quand la vie commence-t-elle? Doit-on considérer le foetus et la femme comme un tout? L'autonomie d'une femme envers son corps doit-elle être limitée? Un père doit-il avoir un droit de veto sur la décision d'avorter d'une femme? A-t-il des droits égaux à ceux de la mère? Comment doit-on définir un fotus? Dans la frénésie et l'urgence, le conflit entre Chantal Daigle et Jean-Guy Tremblay se transformera en un profond débat de société qui marquera le Québec de l'époque. 